\title{
A SIMPLIFIED FORMULATION FOR STRESS AND TRACTION BOUNDARY IN- TEGRAL EQUATIONS USING THE TANGENTIAL DIFFERENTIAL OPERATOR FOR PLATE BENDING INCLUDING THE SHEAR DEFORMATION EFFECT
}

\author{
L. Palermo Jr.
}

Department of Structural, Faculty of Civil Engineering, Architecture and Urban Design, State University of Campinas, Brazil (leandro@fec.unicamp.br)

\begin{abstract}
The existence of strong singularities in the fundamental solution kernels of BIEs for stresses at boundary points and for traction forces requires additional care in numerical implementations with respect to that employed for the displacement BIE. The use of the tangential differential operator (TDO) in conjunction with the integration by parts is one way to reduce the order of strong singularities in these fundamental solution kernels. Stress and traction BIEs using the TDO for plate bending models including the shear deformation effect are studied in this work. A Kelvin-type fundamental solution is the main requirement for applying the TDO in conjunction with integration by parts to reduce the singularities. The TDO and integration by parts were applied to all fundamental solution kernels involving the multiplication of generalized displacements in traction BIEs to reduce the singularities, and the resulting kernels were combinations of those from the displacement BIE, i.e. the numerical implementation requires the same effort employed for displacement BIEs. Plate problems were solved with traction BIEs employing the TDO instead of the displacement BIEs to the behavior evaluation with singularity reduced.
\end{abstract}

Keywords: Reissner plate, tangential operatior, traction equation.

\section{INTRODUCTION}

Distributed shear, bending and twisting moments required in plate bending analyses are computed with stress BIEs. The differentiation in the fundamental solution kernels of displacement BIE, to obtain BIEs for stresses, increases the order of singularities. Strong singularities appear in fundamental solution kernels of stress BIE when values at boundary points are required as well as in those of traction BIE because the equations are related according to the Cauchy formula. The collocation point position and the strategy to treat improper integrals are the essential features studied in numerical implementations for traction or stress BIE in plate bending or in elasticity $[1,2]$. The use of the tangential differential operator (TDO) in conjunction with the integration by parts is a way to reduce the order of singularities in stress or traction BIE when Kelvin type fundamental solutions are used. Kupradze first presented an application using the TDO [3] and Sladek presented some boundary element formulations 
with the TDO [4]. Regularized boundary element formulations employing TDO for potential and elasticity problems, including those for fracture mechanics problems, were presented by Bonnet [5]. Plane problems of linear fracture mechanics were analyzed with the dual boundary element method using the TDO in the traction BIE [6] and a traction BIE formulation with TDO for meshes using non-conformal interpolations in three dimensional problems was proposed [7].

A formulation for traction BIE in plate bending considering the shear deformation effect and using the TDO was proposed [8]. The existence of two tensors in the plate bending equilibrium carries to a second formulation for the traction BIE [9]. In the first formulation, the strong singularity was reduced whereas the other kernels remained unchanged. In the second formulation, all fundamental solution kernels multiplying generalized displacements had their singularities reduced with the TDO and the resulting kernels were combinations of those from the displacement BIEs. In spite of the solutions in the boundary element method usually employ the displacement BIE, plate bending problems were solved with both traction BIEs with the TDO for a better evaluation on their behavior [9]. Similar results were obtained in both formulations, which had isoparametric linear elements in the numerical implementation and employed one collocation point per element in conformal interpolations or two points per element in non-conformal interpolations. The number of collocation points in each element was defined in the code according to the last node condition, i.e. elements with discontinuity at the first node had one collocation point to avoid the solution of an overdetermined linear system of equations. Overdetermined linear system of equations were analyzed in a strategy of using the number of collocation points in each element always equal to the number of nodes regardless the interpolation adopted [10]. The results obtained from the least square solution presented accuracy similar to the regular solution using a square matrix and until with the double number of nodes. Asymmetry issues in conformal interpolations, which have been discussed for traction BIEs with the TDO [9] were fixed in that strategy even using linear elements [10].

According to those former studies on traction BIEs with TDO for plate bending, the second formulation can be considered the simplest BIE for tractions without presenting issues or a reduction on the required accuracy with reference to the well-known formulation with the strong singularity. The explicit relations of the fundamental solution kernels in the second formulation with reference to those in the displacement BIE are presented in this study and the main results representing the formulation behavior are included in this paper when a regular or an overdetermined system of equations are used.

\section{TRACTION BOUNDARY INTEGRAL EQUATION USING THE TANGENTIAL DIFFERENTIAL OPERATOR}

The equilibrium equations for an infinitesimal plate element under a transverse distributed loading $\mathrm{q}\left(\mathrm{x}_{\mathrm{i}}\right)$ are first written with Latin indices considering the values $\{1,2$ and 3$\}$ and Greek indices considering the values $\{1,2\}$ :

$$
M_{\alpha \beta, \beta}-Q_{\alpha}=0
$$




$$
Q_{\alpha, \alpha}+q=0
$$

The plate has a uniform thickness $\mathrm{h}, \mathrm{D}$ is the flexural rigidity, $v$ is the Poisson ratio. A unified notation for Reissner and Mindlin models [10] is used in the following constitutive relations, where $\mathrm{w}$ is the deflection, $\psi_{\alpha}$ is the plate rotation in the direction $\alpha$, and $\delta_{\alpha \beta}$ is the Kronecker delta.

$$
\begin{gathered}
M_{\alpha \beta}=D \frac{(1-v)}{2}\left(\psi_{\alpha, \beta}+\psi_{\beta, \alpha}+\frac{2 v}{1-v} \psi_{\gamma, \gamma} \delta_{\mathrm{a} \beta}\right)+\delta_{\alpha \beta} q R E \\
Q_{\alpha}=D \frac{(1-v)}{2} \lambda^{2}\left(\psi_{\alpha}+w_{, \alpha}\right) \\
\lambda^{2}=12 \frac{\kappa^{2}}{h^{2}} ; \quad R E=\frac{v}{\lambda^{2}(1-v)}
\end{gathered}
$$

The product qRE in Equation (3) corresponds to the linearly weighted average effect of the normal stress component in the thickness direction and should be considered in Reissner's model but not in Mindlin's model, in which it should be considered to be null. The shear parameter $\kappa^{2}$ is equal to $5 / 6$ and $\pi^{2} / 12$ for the Reissner and the Mindlin model, respectively. A unified displacement BIE for these models can be written in terms of the generalized displacements and tractions presented in [11].

$$
\begin{gathered}
c_{i j}\left(x^{\prime}\right) u_{j}\left(x^{\prime}\right)+\int_{\Gamma}\left[T_{i j}\left(x^{\prime}, x\right) u_{j}(x)-U_{i j}\left(x^{\prime}, x\right) t_{j}(x)\right] d \Gamma(x) \\
=\iint_{\Omega}\left[U_{i 3}\left(x^{\prime}, X\right)-U_{i \alpha, \alpha}\left(x^{\prime}, X\right) R E\right] q(X) d \Omega(X)
\end{gathered}
$$

$\mathrm{u}_{\alpha}$ and $\mathrm{u}_{3}$ is equal to $\psi_{\alpha}$ and $\mathrm{w}$, respectively, $\mathrm{t}_{\beta}$ is the product $\mathrm{M}_{\alpha \beta} \cdot \mathrm{n}_{\alpha}$, and $\mathrm{t}_{3}$ is the product $\mathrm{Q}_{\alpha} \cdot \mathrm{n}_{\alpha} \cdot \mathrm{U}_{\mathrm{ij}}$ represents the rotation $(\mathrm{j}=1,2)$ or the deflection $(\mathrm{j}=3)$ arising from a unit couple $(\mathrm{i}=1,2)$ or a unit point force $(\mathrm{i}=3)$, and $\mathrm{c}_{\mathrm{ij}}$ is an element of the matrix $\mathrm{C}$ related to the collocation point position that makes the diagonal matrix elements equal to one for internal collocation points or equal to 0.5 for collocation points on a smooth boundary. The integrand of the domain integral in Equation (5) contains the RE factor, which should be considered null for analyses using the Mindlin model.

The result for the deflection gradient BIE can be written using differentiation in terms of the coordinate of the field point with the direction cosines of the outward normal at the field point written off the differential operator in the notation [9]. 


$$
\begin{aligned}
u_{3, \gamma}\left(X^{\prime}\right)=\int_{\Gamma} & \left\{n_{\alpha}(x) \frac{\partial}{\partial x_{\gamma}}\left[M_{3 \alpha \beta}\left(X^{\prime}, x\right)\right] u_{\beta}(x)+n_{\beta}(x) \frac{\partial}{\partial x_{\gamma}}\left[Q_{3 \beta}\left(X^{\prime}, x\right)\right] u_{3}(x)\right. \\
& \left.-\frac{\partial}{\partial x_{\gamma}}\left[U_{3 \beta}\left(X^{\prime}, x\right)\right] t_{\beta}(x)-\frac{\partial}{\partial x_{\gamma}}\left[U_{33}\left(X^{\prime}, x\right)\right] t_{3}(x)\right\} d \Gamma(x) \\
& -\iint_{\Omega} \frac{\partial}{\partial X_{\gamma}}\left[U_{33}\left(X^{\prime}, X\right)-U_{3 \alpha, \alpha}\left(X^{\prime}, X\right) R E\right] q(X) d \Omega(X)
\end{aligned}
$$

The TDO is introduced in the fundamental solution kernel with the strong singularity when the field point approaches the collocation point, which is $\mathrm{Q}_{3 \beta, \gamma}$. The fundamental solution for a unit point force ( $i=3$ ) is related to the equilibrium Equation (2) in the absence of the transverse distributed load $q$ and is a Kelvin-type solution. $Q_{3 \beta, \beta}$ is equal to zero outside the source point because of the singular behavior of the Kelvin-type solution. The result for Equation (6) with the TDO is next written after integration by parts to reduce the order of the singularity in the fundamental solution kernel where the TDO was introduced, which changed the application of the TDO to $\mathrm{u}_{3}[9]$ :

$$
\begin{aligned}
u_{3, \gamma}\left(X^{\prime}\right) & =\int_{\Gamma}\left\{n_{\alpha}(x) \frac{\partial}{\partial x_{\gamma}}\left[M_{3 \alpha \beta}\left(X^{\prime}, x\right)\right] u_{\beta}(x)+Q_{3 \beta}\left(X^{\prime}, x\right) D_{\gamma \beta}\left[u_{3}(x)\right]\right. \\
& \left.-\frac{\partial}{\partial x_{\gamma}}\left[U_{3 \beta}\left(X^{\prime}, x\right)\right] t_{\beta}(x)-\frac{\partial}{\partial x_{\gamma}}\left[U_{33}\left(X^{\prime}, x\right)\right] t_{3}(x)\right\} d \Gamma(x) \\
& -\iint_{\Omega} \frac{\partial}{\partial X_{\gamma}}\left[U_{33}\left(X^{\prime}, X\right)-U_{3 \alpha, \alpha}\left(X^{\prime}, X\right) R E\right] q(X) d \Omega(X)
\end{aligned}
$$

$\mathrm{D}_{\mathrm{bm}}(\mathrm{)})$ is the tangential differential operator, which has the following definition:

$$
D_{b m}[f(y)]=n_{b}(y) f_{, m}(y)-n_{m}(y) f_{, b}(y)
$$

The algebraic manipulation required to introduce the TDO in the BIE for a rotation gradient at an internal point is similar but not equal to that shown for a deflection gradient because the equilibrium Equation (1) is used, in which the out-of-plane stress (shears) tensor is related to the in-plane stress tensor (the bending and twisting moments) [9]. The final expression for the rotation gradient is the following: 


$$
\begin{aligned}
u_{3, \gamma}\left(X^{\prime}\right) & =\int_{\Gamma}\left\{n_{\alpha}(x) \frac{\partial}{\partial x_{\gamma}}\left[M_{3 \alpha \beta}\left(X^{\prime}, x\right)\right] u_{\beta}(x)+Q_{3 \beta}\left(X^{\prime}, x\right) D_{\gamma \beta}\left[u_{3}(x)\right]\right. \\
& \left.-\frac{\partial}{\partial x_{\gamma}}\left[U_{3 \beta}\left(X^{\prime}, x\right)\right] t_{\beta}(x)-\frac{\partial}{\partial x_{\gamma}}\left[U_{33}\left(X^{\prime}, x\right)\right] t_{3}(x)\right\} d \Gamma(x) \\
& -\iint_{\Omega} \frac{\partial}{\partial X_{\gamma}}\left[U_{33}\left(X^{\prime}, X\right)-U_{3 \alpha, \alpha}\left(X^{\prime}, X\right) R E\right] q(X) d \Omega(X)
\end{aligned}
$$

The TDO was introduced in the fundamental solution kernels to reduce strong singularities; thus, only $1 / \mathrm{r}$ singularities or logarithmic singularities remain. An interesting feature in formulations using the TDO in BIEs for elasticity [6,7] is the direct relationship between fundamental solution kernels in the stress and displacement BIEs, i.e., the fundamental solution kernels of the stress BIE are combinations of those from the displacement BIE. A direct relationship with the displacement BIE can be obtained in the BIE for gradients when the TDO and integration by parts are introduced in the remaining kernels multiplying generalized displacements [9]. The BIE for the distributed shear at internal points can be obtained using the constitutive Equation (4) together with Equations (5) and (7) whereas those BIEs for bending and twisting moments at internal points are obtained with the constitutive Equation (3) together with equation (8). The final expressions for $\mathrm{Q}_{\gamma}$ and $\mathrm{M}_{\alpha \beta}$ containing the direct relationship with displacement BIE are as follows, [9]:

$$
\begin{aligned}
& Q_{\gamma}\left(X^{\prime}\right)=D \frac{(1-v)}{2} \lambda^{2}\left(\int _ { \Gamma } \left\{M_{3 \alpha \beta}\left(X^{\prime}, x\right) D_{\gamma \alpha}\left[u_{\beta}(x)\right]\right.\right. \\
& +\left[n_{\gamma}(x) Q_{3 \beta}\left(X^{\prime}, x\right)-T_{\gamma \beta}\left(X^{\prime}, x\right)\right] u_{\beta}(x)+Q_{3 \beta}\left(X^{\prime}, x\right) D_{\gamma \beta}\left[u_{3}(x)\right] \\
& \left.\left.-T_{\gamma 3}\left(X^{\prime}, x\right) u_{3}(x)\right\} d \Gamma(x)\right) \\
& +\int_{\Gamma}\left[Q_{\beta \gamma}\left(X^{\prime}, x\right) t_{\beta}(x)-Q_{3 \gamma}\left(X^{\prime}, x\right) t_{3}(x)\right] d \Gamma(x) \\
& -\iint_{\Omega} q(X) Q_{3 \gamma}\left(X^{\prime}, X\right) d \Omega(X)
\end{aligned}
$$




$$
\begin{aligned}
& M_{\alpha \beta}\left(X^{\prime}\right)=C_{\alpha \beta \rho \gamma}\left(\int _ { \Gamma } \left\{M_{\rho \eta \tau}\left(X^{\prime}, x\right) D_{\gamma \eta}\left[u_{\tau}(x)\right]+n_{\gamma}(x) Q_{\rho \tau}\left(X^{\prime}, x\right) u_{\tau}(x)\right.\right. \\
& \left.\left.+Q_{\rho \tau}\left(X^{\prime}, x\right) D_{\gamma \tau}\left[u_{3}(x)\right]\right\} d \Gamma(x)\right) \\
& +\int_{\Gamma}\left[M_{3 \alpha \beta}\left(X^{\prime}, x\right) t_{3}(x)-M_{\gamma \alpha \beta}\left(X^{\prime}, x\right) t_{\gamma}(x)\right] d \Gamma(x) \\
& +\iint_{\Omega} q(X) M_{3 \alpha \beta}\left(X^{\prime}, X\right) d \Omega(X) \\
& +R E \iint_{\Omega} q(X) \frac{\partial}{\partial X_{\gamma}}\left[M_{\gamma \alpha \beta}\left(X^{\prime}, X\right)\right] d \Omega(X)+R E \delta_{\alpha \beta} q\left(X^{\prime}\right)
\end{aligned}
$$

With

$$
C_{\alpha \beta \rho \gamma}=D \frac{(1-v)}{2}\left(\delta_{\alpha \rho} \delta_{\beta \gamma}+\delta_{\alpha \gamma} \delta_{\beta \rho}+\frac{2 v}{1-v} \delta_{\alpha \beta} \delta_{\rho \gamma}\right)
$$

The BIE for stresses at a boundary point can be obtained as the limiting form of the corresponding BIE at an external point $\mathrm{X}^{\prime \prime}$ when it tends to the boundary $\Gamma$ at $\mathrm{x}^{\prime}$, which is assumed to be on a smooth boundary to satisfy the continuity requirements. The BIEs for generalized tractions are obtained from the stress BIEs using the Cauchy formula, i.e., the distributed shear tensor and the moment tensor at the collocation point on the boundary point $\mathrm{x}^{\prime}$ are multiplied by the direction cosines of the outward normal at this point ( $\left.\mathrm{n}^{\prime}\right)$ [9]: 


$$
\begin{aligned}
& \frac{1}{2} t_{3}\left(x^{\prime}\right)=n_{\gamma}^{\prime}\left(x^{\prime}\right) D \frac{(1-v)}{2} \lambda^{2}\left(\int _ { \Gamma } \left\{M_{3 \alpha \beta}\left(x^{\prime}, x\right) D_{\tilde{a} \alpha}\left[u_{\beta}(x)\right]\right.\right. \\
& +\left[n_{\gamma}(x) Q_{3 \beta}\left(x^{\prime}, x\right)-T_{\gamma \beta}\left(x^{\prime}, x\right)\right] u_{\beta}(x)+Q_{3 \beta}\left(x^{\prime}, x\right) D_{\gamma \beta}\left[u_{3}(x)\right] \\
& \left.\left.-T_{\gamma 3}\left(x^{\prime}, x\right) u_{3}(x)\right\} d \Gamma(x)\right) \\
& +n_{\gamma}^{\prime}\left(x^{\prime}\right) \int_{\Gamma}\left[Q_{\beta \gamma}\left(x^{\prime}, x\right) t_{\beta}(x)-Q_{3 \gamma}\left(x^{\prime}, x\right) t_{3}(x)\right] d \Gamma(x) \\
& -n_{\gamma}^{\prime}\left(x^{\prime}\right) \iint_{\Omega} q(X) Q_{3 \gamma}\left(x^{\prime}, X\right) d \Omega(X) \\
& \frac{1}{2} t_{\beta}\left(x^{\prime}\right)=n_{\alpha}^{\prime}\left(x^{\prime}\right) C_{\alpha \beta \rho \gamma}\left(\int _ { \Gamma } \left\{M_{\rho \eta \tau}\left(x^{\prime}, x\right) D_{\gamma \eta}\left[u_{\tau}(x)\right]+n_{\gamma}(x) Q_{\rho \tau}\left(x^{\prime}, x\right) u_{\tau}(x)\right.\right. \\
& \left.\left.+Q_{\rho \tau}\left(x^{\prime}, x\right) D_{\gamma \tau}\left[u_{3}(x)\right]\right\} d \Gamma(x)\right) \\
& +n_{\alpha}^{\prime}\left(x^{\prime}\right) \int_{\Gamma}\left[M_{3 \alpha \beta}\left(x^{\prime}, x\right) t_{3}(x)-M_{\gamma \alpha \beta}\left(x^{\prime}, x\right) t_{\gamma}(x)\right] d \Gamma(x) \\
& +n_{\alpha}^{\prime}\left(x^{\prime}\right) \iint_{\Omega} q(X)\left[M_{3 \alpha \beta}\left(x^{\prime}, X\right)+M_{\gamma \alpha \beta, \gamma}\left(x^{\prime}, X\right) R E\right] d \Omega(X) \\
& +\frac{1}{2} R E n_{\beta}^{\prime}\left(x^{\prime}\right) q\left(x^{\prime}\right)
\end{aligned}
$$

The relation with the tangential derivative in the TDO appears when derivatives in TDO are written with reference to the normal (n) and tangent $(\mathrm{t})$ directions to the boundary and final result is given by [9]:

$$
D_{\alpha \beta}[f(y)]=e_{3 \alpha \beta} \frac{\partial f(y)}{\partial t}
$$

$\mathrm{e}_{\mathrm{i} \beta \gamma}$ is the permutation symbol.

The formulation for traction BIE is next written using the relation with tangent derivative in the TDO. It is important to note the application of the TDO on displacements corre- 
sponds to a local derivative in the boundary element formulation.

$$
\begin{aligned}
& \frac{1}{2} t_{3}\left(x^{\prime}\right)=n_{\gamma}^{\prime}\left(x^{\prime}\right) D \frac{(1-v)}{2} \lambda^{2}\left(\int _ { \Gamma } \left\{M_{3 \alpha \beta}\left(x^{\prime}, x\right) e_{3 \gamma \alpha} \frac{\partial\left[u_{\beta}(x)\right]}{\partial t}\right.\right. \\
& +\left[n_{\gamma}(x) Q_{3 \beta}\left(x^{\prime}, x\right)-M_{\gamma \alpha \beta}\left(x^{\prime}, x\right) n_{\alpha}(x)\right] u_{\beta}(x) \\
& \left.\left.+Q_{3 \beta}\left(x^{\prime}, x\right) e_{3 \gamma \beta} \frac{\partial\left[u_{3}(x)\right]}{\partial t}-Q_{\gamma \beta}\left(x^{\prime}, x\right) n_{\beta}(x) u_{3}(x)\right\} d \Gamma(x)\right) \\
& +n_{\gamma}^{\prime}\left(x^{\prime}\right) \int_{\Gamma}\left[Q_{\beta \gamma}\left(x^{\prime}, x\right) t_{\beta}(x)-Q_{3 \gamma}\left(x^{\prime}, x\right) t_{3}(x)\right] d \Gamma(x) \\
& -n_{\gamma}^{\prime}\left(x^{\prime}\right) \iint_{\Omega} q(X) Q_{3 \gamma}\left(x^{\prime}, X\right) d \Omega(X) \\
& \frac{1}{2} t_{\beta}\left(x^{\prime}\right)=n_{\alpha}^{\prime}\left(x^{\prime}\right) C_{\alpha \beta \rho \gamma}\left(\int _ { \Gamma } \left\{M_{\rho \eta \tau}\left(x^{\prime}, x\right) e_{3 \gamma \eta} \frac{\partial\left[u_{\beta}(x)\right]}{\partial t}+n_{\gamma}(x) Q_{\rho \tau}\left(x^{\prime}, x\right) u_{\tau}(x)\right.\right. \\
& \left.\left.+Q_{\rho \tau}\left(x^{\prime}, x\right) e_{3 \gamma \tau} \frac{\partial\left[u_{3}(x)\right]}{\partial t}\right\} d \Gamma(x)\right) \\
& +n_{\alpha}^{\prime}\left(x^{\prime}\right) \int_{\Gamma}\left[M_{3 \alpha \beta}\left(x^{\prime}, x\right) t_{3}(x)-M_{\gamma \alpha \beta}\left(x^{\prime}, x\right) t_{\gamma}(x)\right] d \Gamma(x) \\
& +n_{\alpha}^{\prime}\left(x^{\prime}\right) \iint_{\Omega} q(X)\left[M_{3 \alpha \beta}\left(x^{\prime}, X\right)+M_{\gamma \alpha \beta, \gamma}\left(x^{\prime}, X\right) R E\right] d \Omega(X) \\
& +\frac{1}{2} R E n_{\beta}^{\prime}\left(x^{\prime}\right) q\left(x^{\prime}\right)
\end{aligned}
$$

The displacement BIE is written next in the extended notation (not in the Weeën's notation presented in Equation 5): 


$$
\begin{aligned}
& \frac{1}{2} u_{3}\left(x^{\prime}\right)=\int_{\Gamma}\left[-M_{3 \alpha \beta}\left(x^{\prime}, x\right) n_{\alpha}(x) u_{\beta}(x)-Q_{3 \beta}\left(x^{\prime}, x\right) n_{\beta}(x) u_{3}(x)+U_{3 \beta}\left(x^{\prime}, x\right) t_{\beta}(x)\right. \\
& \left.+U_{33}\left(x^{\prime}, x\right) t_{3}(x)\right] d \Gamma(x) \\
& +\iint_{\Omega}\left[U_{33}\left(x^{\prime}, X\right)-U_{3 \alpha, \alpha}\left(x^{\prime}, X\right) R E\right] q(X) d \Omega(X) \\
& \frac{1}{2} u_{\rho}\left(x^{\prime}\right)=\int_{\Gamma}\left\{-M_{\rho \alpha \beta}\left(x^{\prime}, x\right) n_{\alpha}(x) u_{\beta}(x)-Q_{\rho \beta}\left(x^{\prime}, x\right) n_{\beta}(x) u_{3}(x)+U_{\rho \beta}\left(x^{\prime}, x\right) t_{\beta}(x)\right. \\
& \left.+U_{\rho 3}\left(x^{\prime}, x\right) t_{3}(x)\right\} d \Gamma(x) \\
& +\iint_{\Omega}\left[U_{\rho 3}\left(x^{\prime}, X\right)-U_{\rho \alpha, \alpha}\left(x^{\prime}, X\right) R E\right] q(X) d \Omega(X)
\end{aligned}
$$

The comparison between Equations (14) to (17) shows, explicitly, the kernels in traction BIEs are combinations of those in displacement BIEs.

\section{NUMERICAL IMPLEMENTATION}

The generalized displacements and their derivatives were assumed to be continuous along the boundary in the development to obtain the traction BIEs using TDO. Additional terms in Equations (11) and (12) have to be introduced when non-conformal interpolations are used and the corresponding equations are given by [9]:

$$
\begin{aligned}
& \frac{1}{2} t_{3}\left(x^{\prime}\right)=(\text { terms on RHS of Equation 11) } \\
& \qquad \begin{array}{l}
\quad+n_{\gamma}^{\prime}\left(x^{\prime}\right) D \frac{(1-v)}{2} \lambda^{2}\left(\left[e_{3 \alpha \gamma} M_{3 \alpha \beta}\left(x^{\prime}, x\right) u_{\beta}(x)\right]_{0}^{\Gamma}\right. \\
\left.\quad+\left[e_{3 \beta \gamma} Q_{3 \beta}\left(x^{\prime}, x\right) u_{3}(x)\right]_{0}^{\Gamma}\right)
\end{array} \\
& \begin{array}{l}
\frac{1}{2} t_{\beta}\left(x^{\prime}\right)=(\text { terms on RHS of Equation } 12) \\
\quad+n_{\alpha}^{\prime}\left(x^{\prime}\right) C_{\alpha \beta \rho \gamma}\left(\left[e_{3 \eta \gamma} M_{\rho \eta \tau}\left(x^{\prime}, x\right) u_{\tau}(x)\right]_{0}^{\Gamma}+\left[e_{3 \tau \gamma} Q_{\rho \tau}\left(x^{\prime}, x\right) u_{3}(x)\right]_{0}^{\Gamma}\right)
\end{array}
\end{aligned}
$$


The nodes of the boundary elements were always fixed at ends of each element, and two nodes with same geometrical coordinates were considered in each discontinuity point. The collocation points were placed on boundary element nodes or shifted to the interior of boundary elements, always along the boundary line, according to the BIE and interpolation types.

The continuity for displacements functions at the collocation point position $\left(\mathrm{x}^{\prime}\right)$ is required in displacement BIE (Equation 5) whereas the continuity for derivatives of displacements functions at the collocation point position is required for the traction BIEs, i.e., Equations (11) and (12). The collocation points for the displacement BIE were positioned at element nodes in conformal interpolations and shifted to the interior of boundary elements in the case of non-conformal interpolations in the results shown next. The collocation points in traction BIE were always positioned inside boundary elements for both interpolation types.

Generalized displacements and tractions were mapped in elements with linear shape functions, and constant values were obtained for mapping function derivatives used in TDO [9]. The positions of the collocation points inside the boundary element $\left(\xi^{\prime}\right)$, in the range ($1,1)$, were i) $\xi^{\prime}=-0.67$ in conformal interpolations for the traction BIE and ii) $\xi^{\prime}=-0.67$ and $\xi^{\prime}=+0.67$ in non-conformal interpolations for displacement and traction BIEs.

The implementation for traction BIEs considered one collocation point per element in conformal interpolations and two points per element in non-conformal interpolations to obtain a regular system of equations. Two collocation points per element were used regardless the interpolation type adopted when the overdetermined system of equations was obtained. The collocation points positions $\left(\xi^{\prime}\right)$, in the boundary line, were \pm 0.67 , in the range $(-1,1)$, for continuous or discontinuous elements.

Analytical expressions were used to evaluate singular integrals by applying the Cauchy principal value and the Gauss-Legendre scheme used in regular integrals. To yield analytical expressions, an expansion for small arguments was considered for terms containing modified Bessel functions with real arguments $\left(\mathrm{K}_{0}, \mathrm{~K}_{1}\right)$ when the analysis of the singularity around the origin was considered [12]. The use of the tangent derivative in the TDO requires the Jacobian $(\mathrm{J})$ of the coordinate transformation in the numerical implementation, and the final expression to be integrated is thereby simplified [9]. The diagonal terms of the matrices were obtained directly using the collocation point position in the element and the mapping function. No rigid body conditions were used to obtain the diagonal terms for the displacement or traction BIEs.

The problems were solved with the replacement of the displacement BIE in the boundary element method with traction BIEs to evaluate the behavior of traction BIEs using the TDO. There were written some FORTRAN codes for formulations of traction BIE (a code to obtain a regular system of equations and another to obtain a overdetermined system of equations) as well as one for displacement BIE, and the use of mixed boundary elements, i.e., boundary elements containing a continuous node at one end and a discontinuous node at the other end was allowed. 


\subsection{Torsion in a cube}

A cube with side lengths equal to $2 \mathrm{a}$, where two opposite faces are free of stress and the other faces are under torsion according to the Saint-Venant hypotheses (free warping), as shown in Figure 1. The deflection (w) and the plate rotation in the direction $\mathrm{y}\left(\psi_{\mathrm{y}}\right)$ were used to introduce torsion according to the prescribed rotation angle $(\varphi)$ :

$$
\psi_{y}=\varphi a ; \quad w=\varphi a y
$$

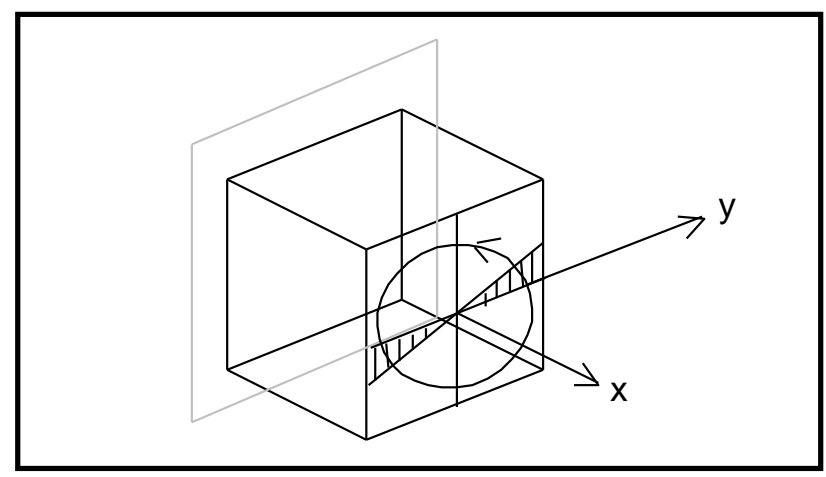

Figure 1 - Torsion of cube

The relative values obtained for plate rotations in the $\mathrm{x}$ direction $\left(\psi_{\mathrm{x}}\right)$, distributed shears $\left(Q_{x}\right)$ in the $x$ direction and twisting moments $\left(M_{x y}\right)$ in Tables 1, 2 and 3, respectively, are used to show the effect of the collocation point position in the traction BIE with the TDO.

Table 1 - Relative values of plate rotations in the normal direction

\begin{tabular}{|c|c|c|c|c|}
\hline$(\mathrm{y} / \mathrm{a})$ & {$[13]$} & $\xi=0.5$ & $\xi=0.67$ & $\xi=0.75$ \\
\hline 1.00 & 1.000 & 1.000 & 1.000 & 1.000 \\
\hline 0.75 & -0.055 & -0.096 & -0.059 & -0.034 \\
\hline 0.50 & -0.387 & -0.429 & -0.391 & -0.366 \\
\hline 0.25 & -0.292 & -0.317 & -0.295 & -0.280 \\
\hline 0.00 & 0.000 & 0.000 & 0.000 & 0.000 \\
\hline
\end{tabular}

Table 2 - Relative values of distributed shears in the normal direction

\begin{tabular}{|c|c|c|c|c|}
\hline$(\mathrm{y} / \mathrm{a})$ & {$[13]$} & $\xi=0.5$ & $\xi=0.67$ & $\xi=0.75$ \\
\hline 1.00 & 1.000 & 1.000 & 1.000 & 1.000 \\
\hline 0.75 & 0.638 & 0.640 & 0.639 & 0.636 \\
\hline 0.50 & 0.377 & 0.377 & 0.377 & 0.375 \\
\hline 0.25 & 0.174 & 0.175 & 0.175 & 0.174 \\
\hline 0.00 & 0.000 & 0.000 & 0.000 & 0.000 \\
\hline
\end{tabular}


Table 3 - Relative values of twisting moments - $\left(\mathrm{M}_{\mathrm{xy}}(\mathrm{y}) / \mathrm{M}_{\mathrm{xy}}(0)\right)$

\begin{tabular}{|c|c|c|c|c|}
\hline (y/a) & {$[13]$} & $\xi=0.5$ & $\xi=0.67$ & $\xi=0.75$ \\
\hline 1.00 & 0.000 & 0.000 & 0.000 & 0.000 \\
\hline 0.75 & 0.485 & 0.487 & 0.486 & 0.485 \\
\hline 0.50 & 0.785 & 0.786 & 0.786 & 0.785 \\
\hline 0.25 & 0.948 & 0.949 & 0.948 & 0.948 \\
\hline 0.00 & 1.000 & 1.000 & 1.000 & 1.000 \\
\hline
\end{tabular}

There was used a mesh with 128 linear discontinuous elements (256 nodes) along the sides to model the complete plate and the values obtained were compared with the Reissner solution for this problem. The greatest differences to the Reissner solution appeared in the results obtained for plate rotations in the normal direction because these values are indirectly related to displacements prescribed.

Table 4 - Relative values for plate rotations in the normal direction, $\left(\psi_{\mathrm{x}}(\mathrm{y}) / \psi_{\mathrm{x}}(\mathrm{a})\right)$

\begin{tabular}{|c|c|c|c|c|c|c|c|c|c|}
\hline \multirow{3}{*}{ y/a } & \multirow{3}{*}{ [13] } & \multicolumn{2}{|c|}{$\begin{array}{c}\text { Discontinuous } \\
\text { elements }\end{array}$} & \multicolumn{6}{|c|}{ Continuous elements } \\
\hline & & \multirow{2}{*}{$\begin{array}{c}128 \mathrm{QE} \\
384 \mathrm{~N} \\
{[4]}\end{array}$} & \multirow{2}{*}{$\begin{array}{c}128 \mathrm{LE} \\
256 \mathrm{~N} \\
{[9]}\end{array}$} & \multicolumn{2}{|c|}{$\begin{array}{c}128 \mathrm{LE} \\
132 \mathrm{~N}\end{array}$} & \multicolumn{2}{|c|}{$\begin{array}{c}192 \mathrm{LE} \\
196 \mathrm{~N}\end{array}$} & \multicolumn{2}{|c|}{$\begin{array}{c}256 \mathrm{LE} \\
132 \mathrm{~N}\end{array}$} \\
\hline & & & & [9] & LSS & Reg. & LSS & Reg. & LSS \\
\hline 1.00 & 1.000 & 1.000 & 1.000 & 1.000 & 1.000 & 1.000 & 1.000 & 1.000 & 1.000 \\
\hline 0.75 & -0.055 & -0.051 & -0.059 & -0.057 & -0.059 & -0.056 & -0.057 & -0.056 & -0.056 \\
\hline 0.50 & -0.387 & -0.382 & -0.391 & -0.389 & -0.391 & -0.388 & -0.389 & -0.387 & -0.388 \\
\hline 0.25 & -0.292 & -0.290 & -0.295 & -0.293 & -0.295 & -0.293 & -0.293 & -0.292 & -0.293 \\
\hline 0.00 & 0.000 & 0.000 & 0.000 & 0.000 & 0.000 & 0.001 & 0.000 & 0.000 & 0.000 \\
\hline-0.25 & -0.292 & -0.290 & -0.295 & -0.297 & -0.295 & -0.294 & -0.293 & -0.293 & -0.293 \\
\hline-0.50 & -0.387 & -0.382 & -0.391 & -0.393 & -0.391 & -0.390 & -0.389 & -0.388 & -0.388 \\
\hline-0.75 & -0.055 & -0.051 & -0.059 & -0.061 & -0.059 & -0.058 & -0.057 & -0.056 & -0.056 \\
\hline-1.00 & 1.000 & 1.000 & 1.000 & 1.000 & 1.000 & 1.000 & 1.000 & 1.000 & 1.000 \\
\hline \multicolumn{2}{|c|}{$\begin{array}{c}\text { Maximum } \\
\text { difference }(\%)\end{array}$} & 7.27 & 7.27 & 10.91 & 7.27 & 5.45 & 3.64 & 1.82 & 1.82 \\
\hline
\end{tabular}

Reg. is regular solution and LSS is linear square solution [10].

Three meshes were used in the complete plate model and 6 Gauss points in the numerical integration to show the behavior of the second formulation for traction BIE with TDO. Two nodes were considered in each corner and conformal interpolations were adopted along the sides for the following meshes: 128 elements (132 nodes), 192 elements (196 nodes) and 256 elements (260 nodes). The results were compared with regular solutions using quadratic elements [1], in which the traction BIE contained the strong singularity and 128 discontinuous elements (384 nodes) were used, as well as using linear elements [9], in which the traction BIEs used the TDO, beyond the Reissner solution [13]. Furthermore, results obtained with the 
same meshes but using the strategy to obtain the overdetermined system of equations were included [10]. These values obtained for plate rotations in the normal direction were used to show the behavior of the formulation. The values for distributed shear and twisting moments were not used, because they are directly related to displacements constrained and the differences to the Reissner solution were extremely low.

\section{CONCLUSIONS}

The use of the TDO requires a Kelvin-type fundamental solution, whereas traction BIE containing a strong singularity is a general formulation that can be applied to all fundamental solution types. Conversely, the numerical implementation of the TDO in the boundary element method is simplified beyond the point of benefiting from singularity reduction [9]. The formulation presented here for traction BIEs with the TDO contains the direct relation of the displacement BIE because the fundamental solution kernels are combinations from those of displacement BIE.

The strategy of using the number of collocation points for traction BIEs equals to the number of nodes in each element regardless the interpolation type adopted, and employing the least square solution, was interesting [10]. The increase in the computation time due to the use of more collocation points and operations of the least square solution can be justified by the increase of the accuracy without increasing the number of degrees of freedom. Furthermore, the benefit of obtaining a symmetric positive definite matrix can be interesting when iterative solutions are required in a problem.

\section{Acknowledgements}

The author is grateful to CNPq support for the development of studies on plates.

\section{REFERENCES}

[1] Rashed YF, Aliabadi MH, Brebbia CA. Hypersingular boundary element formulation for Reissner plates. Int J Solids Struct, 35(18), 2229-49, 1998.

[2] Guiggiani, M. Hypersingular formulation for boundary stresses evaluation. Eng. Anal. Bound. Elem., 14, 169-179, 1994.

[3] Kupradze, V.D., Three-dimensional problems of the mathematical theory of elasticity and thermoelasticity. North Holand, 1979.

[4] Balas, J., Sladek, J, Sladek, V., Stress Analysis by Boundary Element Methods, Elsevier Science Publishers, 1989.

[5] Bonnet, M. Boundary Integral Equation Methods for Solids and Fluids, John Wiley \& Sons Ltd, 1999. 
[6] Palermo Jr., L., Almeida, L.P.C.P.F. and Gonçalves, P.C., The Use of the Tangential Differential Operator in the Dual Boundary Element Equation, Structural Durability \& Health Monitoring, vol.2, no.2, pp.123-130, Tech Science Press, 2006.

[7] Palermo Jr., L., Almeida, L.P.C.P.F., On the Use of the Tangential Differential Operator in the Traction Boundary Integral Equation of the Dual Boundary Element Method for Three Dimensional Problems, ICCES, vol.7, no.2, pp.83-87, 2008.

[8] Palermo Jr., L., Tangential differential operator applied to stress and traction boundary integral equations for plate bending including the shear deformation effect, BEM 33, Editor C.A. Brebbia, 2011.

[9] Palermo Jr., L., The tangential differential operator applied to a stress boundary integral equation for plate bending including the shear deformation effect, Eng Anal Bound Elem, $36,1213-1225,2012$

[10] Palermo Jr., L., A least square solution applied to plate analyses using the traction boundary integral equation, BEM 34, Editor C.A. Brebbia, 2012.

[11] Weeën, F., Application of the boundary integral equation method to Reissner's plate model, International Journal for Numerical Methods in Engineering, vol. 18, 1-10, 1982.

[12] Abramowitz M, Stegun I. Handbook of mathematical functions, New York: Dover Publications; 1972.

[13] Reissner, E., The Effect of Transverse Shear Deformation on the Bending of Elastic Plates, Journal of Applied Mechanics, 1945.

[14] Birkhoff, G, Mac Lane, S., A Survey of modern algebra, $4^{\text {th }}$.ed., Macmillan Publishing Co. Ltd., 1977.

[15] First A. A., Second B. B., "Example of nice winter weather". Int. J. Winter Weat. 7, 813, 2012.

[16] Other A. A., Other B. B., "Example of good quality illustrations". Int. J. Quality Illustr. $7,8-13,2012$. 